\title{
HARD X-RAY PROPERTIES OF CATACLYSMIC VARIABLES
}

\author{
J.H. Swank \\ Laboratory for High Energy Astrophysics \\ NASA/Goddard Space Flight Center \\ Greenbelt, Maryland 20771 U.S.A.
}

The production of hard $X$-rays in accretion onto degenerate dwarfs appears manifested by such sources as the magnetic variables AM Hercul is and 3U0311-227 and the dwarf novae SS Cygni, EX Hydrae and U Geminorum. Upper limits on flux above $2 \mathrm{keV}$ from Ariel 5 (Watson, Sherrington and Jameson 1978) and from the high energy detectors of the HEAO A2 experiment show that only a few others usually exhibit more than $210^{-11}$ ergs $\mathrm{cm}^{-2} \mathrm{~s}^{-1}$ or $\sim 10^{31}$ ergs $\mathrm{s}^{-1}$. However, while there are only a few sources that these experiments could study, their behavior is complex (Swank et al. 1977a; Ricketts, King and Raine 1979; Swank et a1. 1978; Mason, Cordova and Swank 1979). This paper is a brief summary of results for AM Her, EX Hya, SS Cyg and U Gem obtained with the high energy detectors of the HEAO A2 experiment from 2-60 keV and the Solid State Spectrometer Experiment (SSS) with the Monitor Proportional Counter (MPC) on the Einstein Observatory.

Our observations of AM Her in 1975 with 0SO-8 (Swank et al. 1977a) and in 1977 with HEAO A2 (Swank et a1。1977b) were consistent with the $x$-ray source being eclipsed for $\sim 1 / 4$ of a binary orbit. The spectrum appeared as hard as bremsstrahlung with $\mathrm{kT} \sim 100 \mathrm{keV}$. These observations recommended the model in which the $X$-ray source is at one pole of the degenerate dwarf and is eclipsed by the dwarf itself as it synchronously rotates. Our recent observations give a more detailed picture of the light curve and evidence favoring our speculation that albedo from the white dwarf contributes significantly to the hard $X$-ray spectrum.

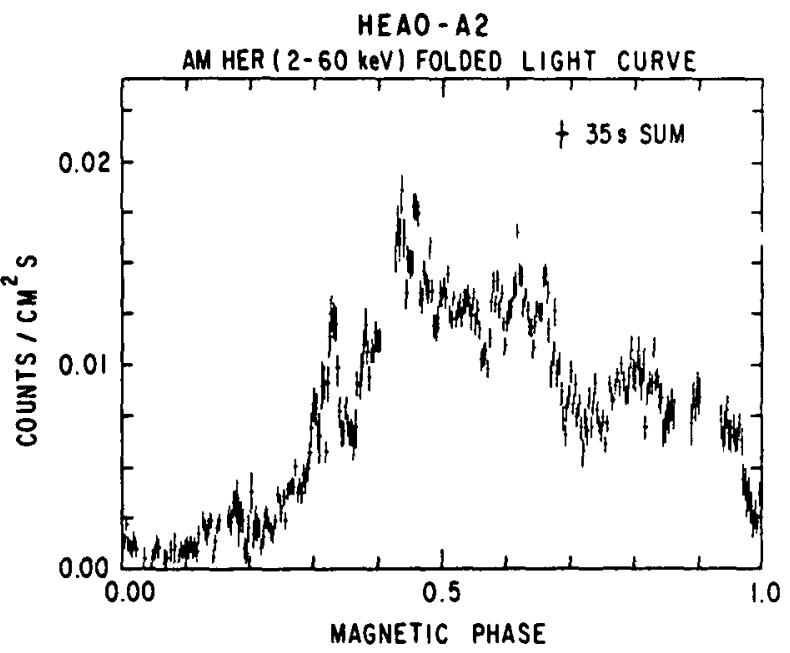

The light curve folded on the binary period for 2 observations $\sim 20$ days apart, each covering $\sim 2 \frac{1}{2}$ binary orbits is shown in Fig. 1. There was not full phase

Fig. 1. Background subtracted data folded with reference to JD2443343.789 on a period of $.128928 \mathrm{~d}$. 
coverage for any one orbit and some of the features represent contributions to only one binary orbit. Examples are the flares of several minutes near phases .32 and .6. Irregular flaring of this sort was always present between phases $.3-.65$. The positive flux for phases $.1-.2$ was present for 3 consecutive orbits but not present 20 days earlier. Variation in the height or angle of an X-ray emitting column or emission from a second pole could produce such a variable feature.

Our observations of dwarf novae cover a range of optical states. Fig. 2 shows a schematic representation of the hard X-ray flux with

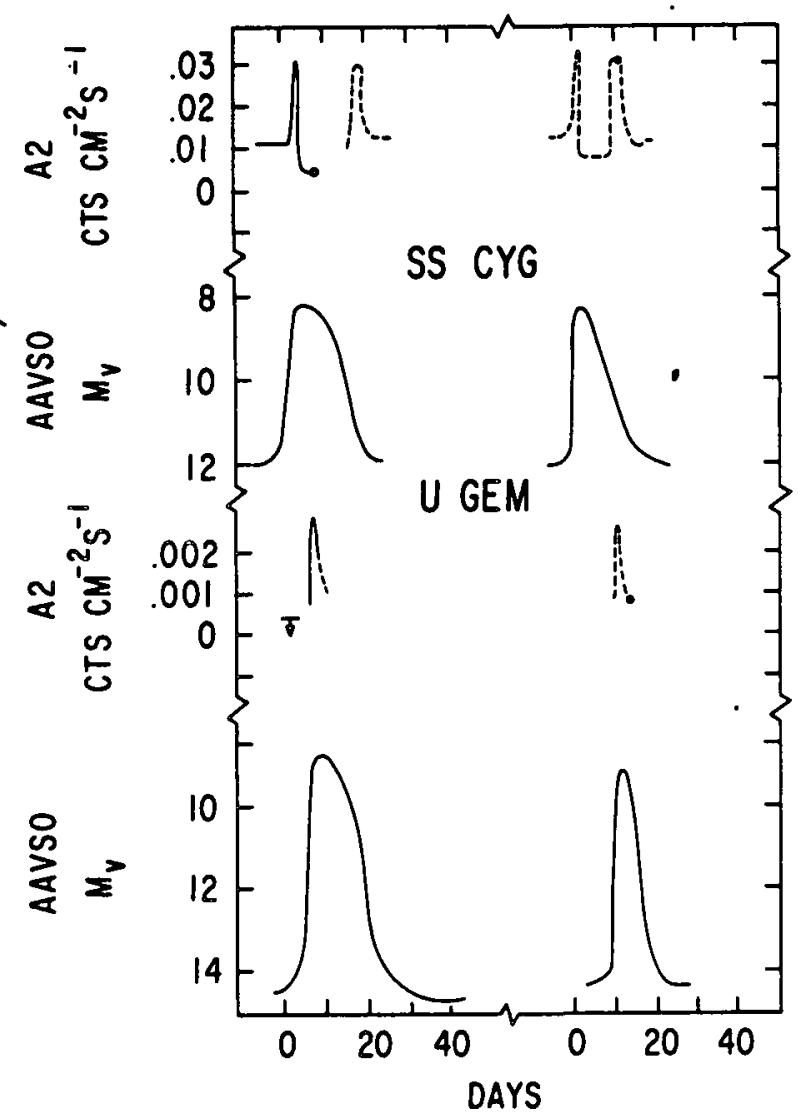

Fig. 2. HEAO A2 light curves in the scan mode $(-)$, and pointed mode $\bullet$; speculated light curves on the basis of other observations (---). For SS Cyg the origins of the time scales are JD 2443668 and 2443832 respectively and for $U$ Gem 2443436 and 2443794. reference to the visual magnitude supplied by the A.A.V.S.O. The fluxes we saw from SS Cyg (Swank et al. 1979) confirm the general behavior traced out by Ricketts, King and Raine (1979), although the maximum $X$-ray flux appeared to coincide with optical maximum. In agreement with the Ariel 5 resuit the flux was found to be as high when the optical magnitude was 11 during the decline of another outburst. There is thus not a unique correspondence between $X$-ray flux and optical magnitude. During scans over U Gem an outburst was discovered in the soft $X$-rays as reported by Mason et a1. (1978). The hard $X$-rays also flared about a day before the soft $X$-rays with a peak flux $1 / 10$ that of SS Cyg at optical max. imum (Swank et al. 1978). A second observation about 2 days after a different maximum found similar hard and soft $x$-ray fluxes, with the soft $X$-rays exhibiting pulsations at $25 \mathrm{~s}$ (Cordova, Chester and Garmire 1979). The temporal behavior suggests similarity to SS Cyg except that there are differences in detail and the maximum $U$ Gem hard flux was just below minimum for SS Cyg.

In all the observations of the dwarf novae the spectra are well fit by thin thermal bremsstrahlung models. Fig. 3 shows two SS Cyg spectra compared with spectra of AM Her obtained with the same detectors. The main SS Cyg flux at high luminosity is $20 \mathrm{keV}$ bremsstrahlung. At a minimum hard $X$-ray luminosity of $4 \times 10^{-11}$ ergs $\mathrm{cm}^{-2} \mathrm{~s}^{-1} \mathrm{kT} \sim 7 \mathrm{keV}$. For $U$ Gem at $1.510^{-11}{\mathrm{ergs} \mathrm{cm}^{-2}}^{-2}$ $\mathrm{s}^{-1}$ we found $\mathrm{kT} \sim 4 \mathrm{keV}$. Errors on $\mathrm{kT}$ are $\sim 1 \mathrm{keV}$. Ricketts, King and Raine (1979) pointed out that during the high mass flow at optical maximum the hard $X$-rays could be absorbed with a cutoff at $10 \mathrm{keV}$ in the incoming material and the energy reemitted as soft $X$-rays. The 


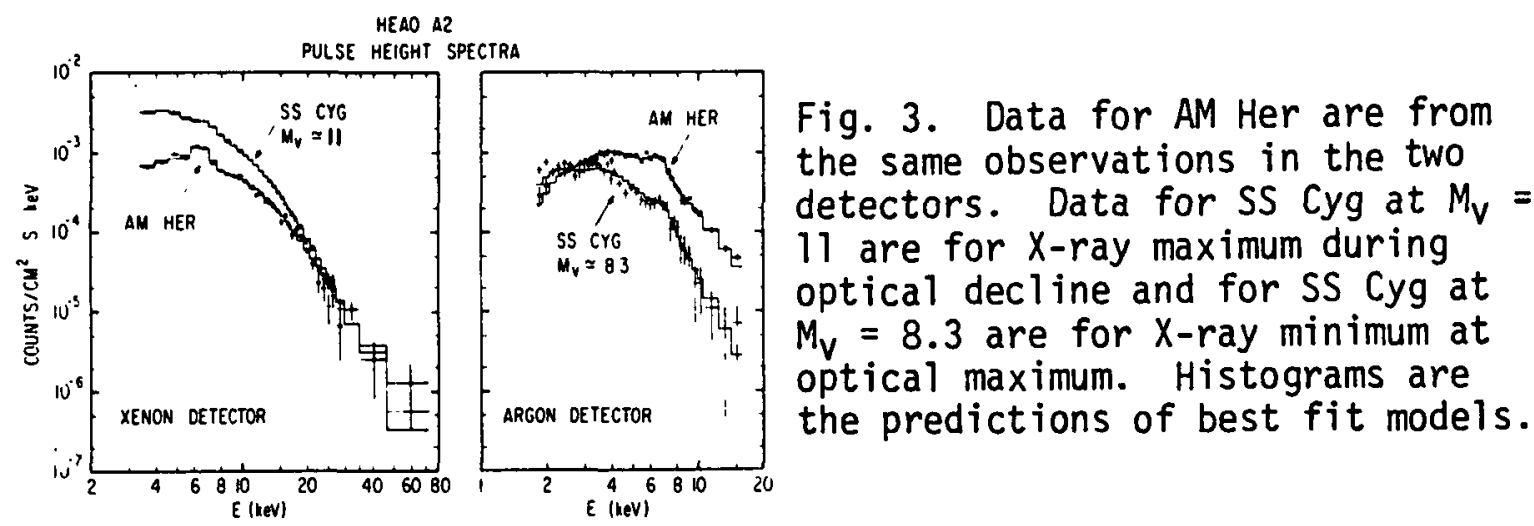

hard $X$-rays that do emerge are not characterized by high absorption. While $U$ Gem has never shown a hard $X$-ray flux comparable to that of SS Cyg, when at optical and soft $X$-ray maximum, the spectra as well as the fluxes are similar.

Our data for AMH Her indicates a harder spectrum than for SS Cyg. The HEAO A2 data for AM Her cannot all be fit to a one temperature model, but it can be fit to an approximation to a model including an albedo. R. Rothschild has combined the simultaneous A2 and A4 data to determine a best fit temperature above $10 \mathrm{keV}$ (Rothschild et al. 1979) of $38 \pm$ $8 \mathrm{keV}$.

The features which can be seen in the pulse height spectra between 6 and $7 \mathrm{keV}$ appear at slightly different energies for AM Her and the dwarf novae: $6.4+.1 \mathrm{keV}$ versus $6.9 \pm .15 \mathrm{keV}$. If the gases are assumed to be in collisional equilibrium, the abundances of Fe implied by the fits are within $50 \%$ of solar for all the dwarf novae, but 8 times too large for AM Her. Thus the line energies and the abundances are consistent in the case of the dwarf novae with thermal emission of gas in collisional equilibrium, while both favor the AM Her features being due to fluorescence.

The lower temperature dwarf novae would not be expected to have so high an albedo or so large a fluorescent line flux. The lines observed in those cases are significant for models of why the temperatures are lower. If the spectra had been degraded in collisions with cooler plasma the line would have been broadened by a few optical depths to scattering.

No pulsations attributable to rotation of a pole or self-eclipses have been found that would substantiate for dwarf novae the model of accretion at magnetic poles. Our limits on pulsed power below a few minutes are from $2 \%$ for SS Cyg to $20 \%$ for $U$ Gem. The 1 imit is $6 \%$ for SS Cyg during the time soft X-ray pulsations were discovered (Cordova et a1. 1979). We reported (Mason, Cordova and Swank 1979) evidence for variability on a scale of 10 minutes in SS Cyg at optical minimum. We have also observed this behavior in SS Cyg at its highest hard $X$-ray flux level. The HEAO A2 observation was syncopated with observations by the SSS and MPC on the Einstein Observatory. Fig. 4 shows a segment of data from the 3 experiments. In some cases the SSS showed flares simultaneously with the MPC. There appears to have been approximate but not exact phase coherence over the $8 \mathrm{hr}$ observation as shown by the folds, with the same reference point, over the period of most significant power. 


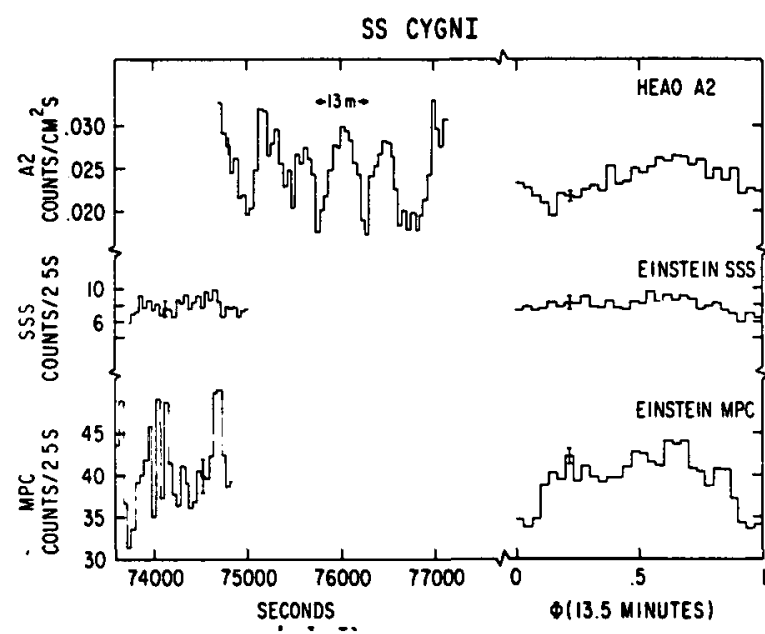

Fig. 4. On the left background subtracted, aspect corrected, light curves for a part of the data. On the right the results of folding all of the data. Typical statistical errors are shown.

In summary, the magnetic variable AM Her and the dwarf novae are similar in having bremsstrahlung spectra of similar luminosity with temperatures for the dwarf novae that can approach that for AM Her and in flickering on a time scale of 5-15 min. However, in general these dwarf novae teniperatures are 2-10 times lower. Only in AM Her is there evidence in the spectrum and eclipses that we see a small spot very close to the white dwarf surface. Most of the hard X-rays in SS Cyg, EX Hya and $U$ Gem are not pulsed and the hard $X$-ray fluxes observed have never been significantly absorbed or degraded.

\section{ACKNOWLEDGMENTS}

The HEAO A2 experiment depended on the A.A.V.S.O. and J. Mattei, Director, not only for the visual light curves, but for notification that outbursts were occurring.

\section{REFERENCES}

Cordova, F.A., Chester, T.J. and Garmire, G.P. 1979, preprint.

Cordova, F.A., Chester, T.J., Tuohy, I.R. and Garmire, G.P. 1979, Ap. J. in press.

Mason, K.O., Lampton, M., Charles, P. and Bowyer, S. 1978, Ap.J. (Letters) 226, L129.

Mason, K., Cordova, F. and Swank, J. 1979 Proceedings of the XXI COSPAR/IAU Symposium in X-ray Astronomy, Pergamon Press Ltd., Oxford.

Ricketts, M.J., King, A.R. and Raine, D.J. 1979, M.N.R.A.S. 186, 233.

Rothschild, R.E. et al. 1979, in preparation.

Swank, J., Lampton, M., Boldt, E., Holt, S. and Serlemitsos, P. 1977a, Ap. J. (Letters) 216, L71.

Swank, J.H., Boldt, E.A., Holt, S.S., Lampton, M., Rothschild, R.E. and Serlemitsos, P.J. 1977b, Bull. A.A.S. 9 , 591.

Swank, J.H., Boldt, E.A., Holt, S.S., Rothschild, R.E. and Serlemitsos, P.J. 1978, Ap. J. (Letters) 226, L133.

Swank, J.H. et al. 1979, in preparation.

Watson, M.G., Sherrington, M.R. and Jameson, R.F. 1978, M.N.R.A.S. 184, 79p. 